\title{
An Analysis of A Single Solar Radio Burst Type III and Type II Coronal Mass Ejections Associated to Solar Flare Event
}

\author{
M. Omar Ali ${ }^{1}$, Z. S. Hamidi ${ }^{1}$, N. N. M. Shariff ${ }^{2}$, C. Monstein ${ }^{3}$ \\ ${ }^{1}$ School of Physics and Material Sciences, Faculty of Sciences, MARA University of Technology, \\ 40450, Shah Alam, Selangor, Malaysia \\ ${ }^{2}$ Academy of Contemporary Islamic Studies (ACIS), MARA University of Technology, 40450, Shah \\ Alam, Selangor, Malaysia \\ ${ }^{3}$ Institute of Astronomy, Wolfgang-Pauli-Strasse 27, Building HIT, Floor J, CH-8093 Zurich, \\ Switzerland \\ E-mail address: zetysh@salam.uitm.edu.my
}

Keywords: Sun; solar burst; type III; radio region; X-ray region; solar flare; active region

\begin{abstract}
Solar radio burst type III and II is the subject matter that we are focusing on because type II and III burst are seem to have relation to each other. The most common of type III burst is called isolated type III burst which is produced by energetic electron from small scale energy release site on the sun and it is ranging from small bright point to large active region. This stage can be considered as a pre-flare stage that could be a signature of electron acceleration. Nevertheless, the most important is that the nonlinear wave-wave interaction which involving interaction of electrostatic electron plasma that called as Langmuir waves active region radio emissions is believed to be a main subject that relevant with a type III burst. In this study, solar radio bursts are observed by using the CALLISTO spectrometer. The log Periodic Dipole Antenna (LPDA) involved in this search over a broad region centered on the Sun and it covered the range of frequency from $45 \mathrm{MHz}-870 \mathrm{MHz}$ and it is connected to the CALLISTO spectrometer. At certain period of time, when the Sun launches billons tones of electrically conducting gas plasma into the space at millions of miles per hours it is assigned that CMEs begin to launches. At this time, the appearance of SRBT III was observed and followed by SRBT II within the time interval of 15 minutes. During flares, large scale of magnetic field structures can be destabilized and be repelled into the interplanetary medium; along with the large mass it contains to form so-called CMEs. Based on the result obtained, the SRBT III is followed by SRBT II which only in short period. During the SRBT II, the solar flare was also appearing and same goes to the CMEs.
\end{abstract}

\section{INTRODUCTION}

Basically the appearance of solar storm such Coronal Mass Ejection (CMEs) and solar flare, solar radio burst can be observed over a broad frequency range. Coronal Mass Ejections (CMEs) are enormous eruptions of plasma and magnetic fields ejected from the sun into interplanetary space, seen by coronagraphs as they move out of their field of view over the course of minutes to hours. CMEs can only be observed by blocking the intense glare of photosphere because their brightness is of order of magnitude of the solar corona. In this study, solar radio burst type III and II is the subject matter that we are focusing on because type II and III burst are seem to have relation to each other. During a flare, large quantities of energy are transferred between the corona and the chromosphere through thermal conduction, non-thermal particle beams, radiation transport and the mass motion. Solar Radio Burst Type III in the IP medium can be grouped into three broad classes representing three different situations of electron beam production and propagation: (i) isolated type III bursts from flares and small-scale energy releases, (ii) complex type III bursts during CMEs, and (iii) type III storms. We discuss them in turn in the next three subsections.

The most common of type III burst is called isolated type III burst which is produced by energetic electron from small scale energy release site on the sun and it is ranging from small bright point to large active region [1,2,3]. It reveals a wave-particle and wave-wave interactions in 
magnetic traps in the solar corona [4]. This burst is still one of the interest burst in order to understand the flare plasma diagnostics in the low corona [5]. Interestingly, the motion follows the predominant magnetic field direction, the apparent speed is a significant fraction of the speed of light. CME interactions were first identified as long - wavelength radio enhancements in the wind in the Wind/WAVES dynamic spectra $[6,7,8]$.

Type II burst are confined to frequency $\sim 150 \mathrm{MHz}$, although occasionally they are observed at higher frequencies. The type II burst are produced by plasma emission mechanism and observed slowly drifting features in the radio dynamic spectrum. Payne-Scott is the one who first discover the slow drifting radio burst (1947) from the records at 200, 100 and $60 \mathrm{MHz}$. It is well known that frequency drift rate of a type II burst is related to the speed of the shock that produces the burst and density gradient in the ambient medium.

The solar radio burst type III solar burst is the most dominant with the solar flare phenomenon was first introduced by Wild in 1963 [9] in the frequency range $500-10 \mathrm{MHz}$ $[10,11,12]$. There are three sub-types of Type III burst that originate in the interplanetary (IP) medium which are (i) isolated Type III bursts from energy system and small-scale energy releases, (ii) a complex Type III bursts during CMEs, and (iii) Type III storms. This stage can be considered as a pre-flare stage that could be a signature of electron acceleration [13]. It is found that $60 \%$ of fast drifts (type III) solar radio bursts are synchronized in time with solar flares [14]. Some evidence showed that type III are generated in a weak-field region comes from the absence or low degree of circular polarization of the bursts [15]. Nevertheless, the most important is that the nonlinear wavewave interaction which involving interaction of electrostatic electron plasma that called as Langmuir waves active region radio emissions is believed to be a main subject that relevant with a type III burst $[16,17,18,19,20]$. It is believed that a beam-plasma system is unstable to the generation of Langmuir waves, which are high frequency plasma waves at the local plasma frequency [21,22]. Type III bursts early in the rise of impulsive solar flares may indicate that open field lines are an essential part of models for energy release by magnetic fields in such flares $[23,24]$. Nevertheless, it is important to analyze in radio and $x$-ray region to understand the distribution of high and low energy [25,26,27,28]. To explain the unique features of what happen during CME, one has to recall the ability of a CME to drive a shock depends not only on its speed, but also on the properties of the medium through which the CME propagates. Further exploration of the relationship between solar burst type II and III with the solar storm such solar flare and CMEs is done to get more information of the evolution of all those phenomenon interactions.

\section{SOLAR FLARE OBSERVATION AND A-CALLISTO SOLAR SPECTROMETER NETWORK}

Solar flare produce copious amount of coherent radio waves, which have been classified for more than 40 years into different classes. Energy during solar flares is used to accelerate particle to high energy. Whereby, electrons will propagate in the solar atmosphere and emitting radio waves when passing the magnetic field. Next, it will collide in the dense lower atmosphere and emit high energy $[29,30,31]$.

Most of the CALLISTO sites focused the frequency $45 \mathrm{MHz}$ to $900 \mathrm{MHz}$ region seems this is the best range with a very minimum of Radio Frequency Interference (RFI). In this study, the data were observed on 27th January 2011 at 12:14 UT till 13:00 UT. The coronagraphs were taken from Solar and Heliospheric Observatory data (SOHO).

In this study, solar radio bursts are observed by using the CALLISTO spectrometer. The log Periodic Dipole Antenna (LPDA) involved in this search over a broad region centered on the Sun and it covered the range of frequency from $45 \mathrm{MHz}-870 \mathrm{MHz}$ and it is connected to the CALLISTO spectrometer [32,33]. Signal from the feed will be fed into the receivers. After that, the signal will be converted to a first intermediate frequency of $37.7 \mathrm{MHz}$ by two local oscillators [31,34,35,36,37]. This antenna covered from $45-870 \mathrm{MHz}[38,39,40,41]$. 
The CALLISTO spectrometer is a low-cost radio spectrometer used to monitor metric and decametric radio bursts, and which has and the named CALLISTO which is inspired from the name of one of the Jupiter's larger moons[42,43,44,45,46]. In this case, we focused the range of $150 \mathrm{MHz}$ till $900 \mathrm{MHz}[47,48,49]$. CALLISTO consist three main components which are the receiver, a linear polarized antenna and control/logging software [50,51]. We have selected the data from the 150 $\mathrm{MHz}$ till $900 \mathrm{MHz}$ region seems this is the best range with a very minimum of Radio Frequency Interference (RFI) $[51,52,53,54,55]$. In this paper, we have focused the study area of solar flares in an X-ray and radio region to evaluate the distribution of high and low energy [38].

\section{RESULTS AND ANALYSIS}

At certain period of time, when the Sun launches billons tones of electrically conducting gas plasma into the space at millions of miles per hours it is assigned that CMEs begin to launches. It is critical when CMEs and the magnetic field which laced together with CMEs' cloud smashed into Earth magnetic field. This is because; they will dump energy into earth magnetic field that can cause magnetic storms. Widespread blackouts by overloading power line equipment will happen due to the storms.

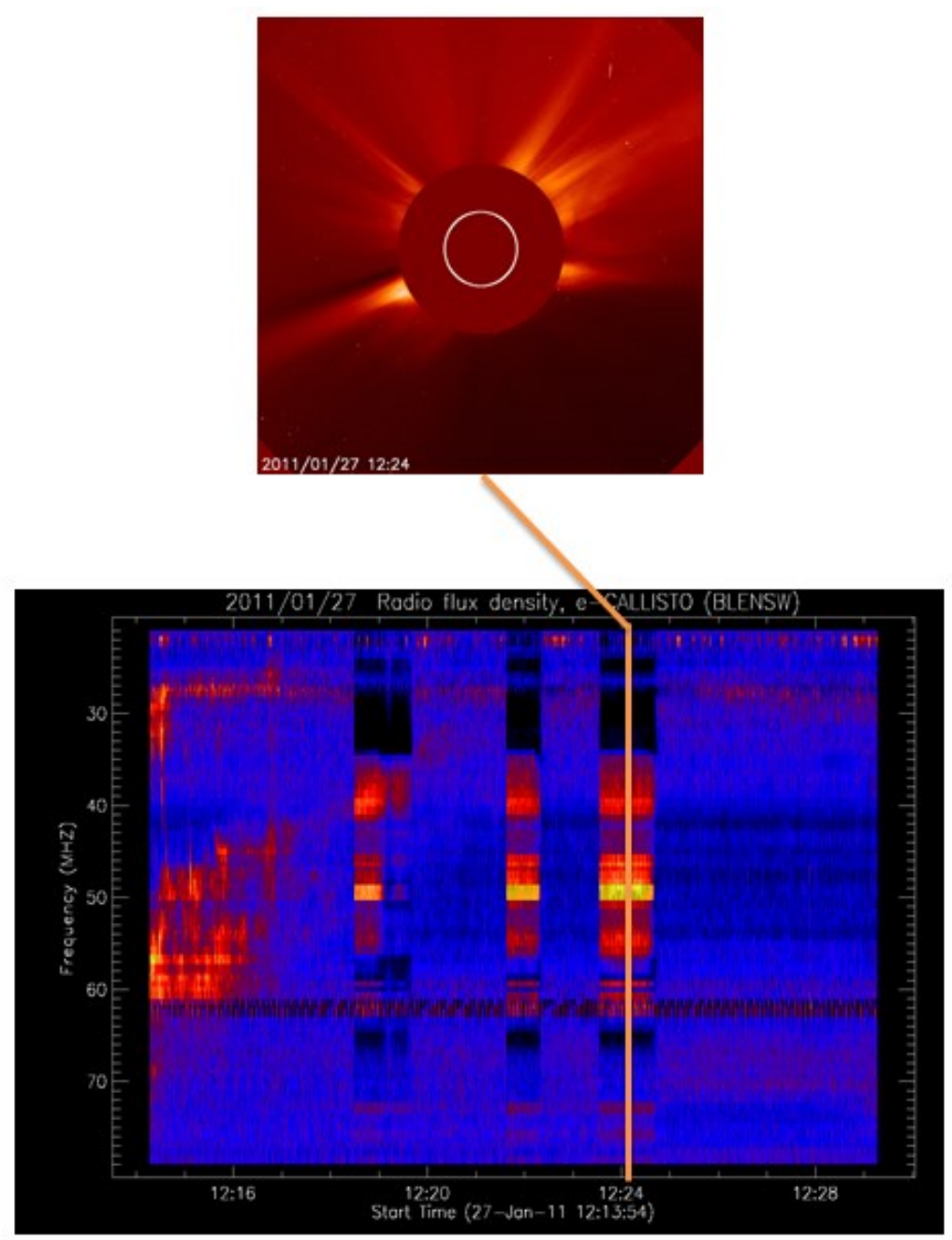

Figure 1: e-Callisto Spectrometer at 12:14 UT - 12:30 UT and LASCO C2 images (Credited to eCallisto and $\mathrm{SOHO})$ 
At this time, the appearance of SRBT III was observed and followed by SRBT II within the time interval of 15 minutes. It was appear at range of $30 \mathrm{MHz}$ to $\mathrm{M} 90 \mathrm{~Hz}$ for about 4 minutes. During the existence of SRBT II, the solar flare also observed. Solar flares are classified according to their X-ray brightness in the wavelength 1-8 Angstrom. Basically, there are three categories of flares, which are X-class flares, M-class flares and C-class flares. X-class flares are the brightest, followed by M-class flare and C-class flare.

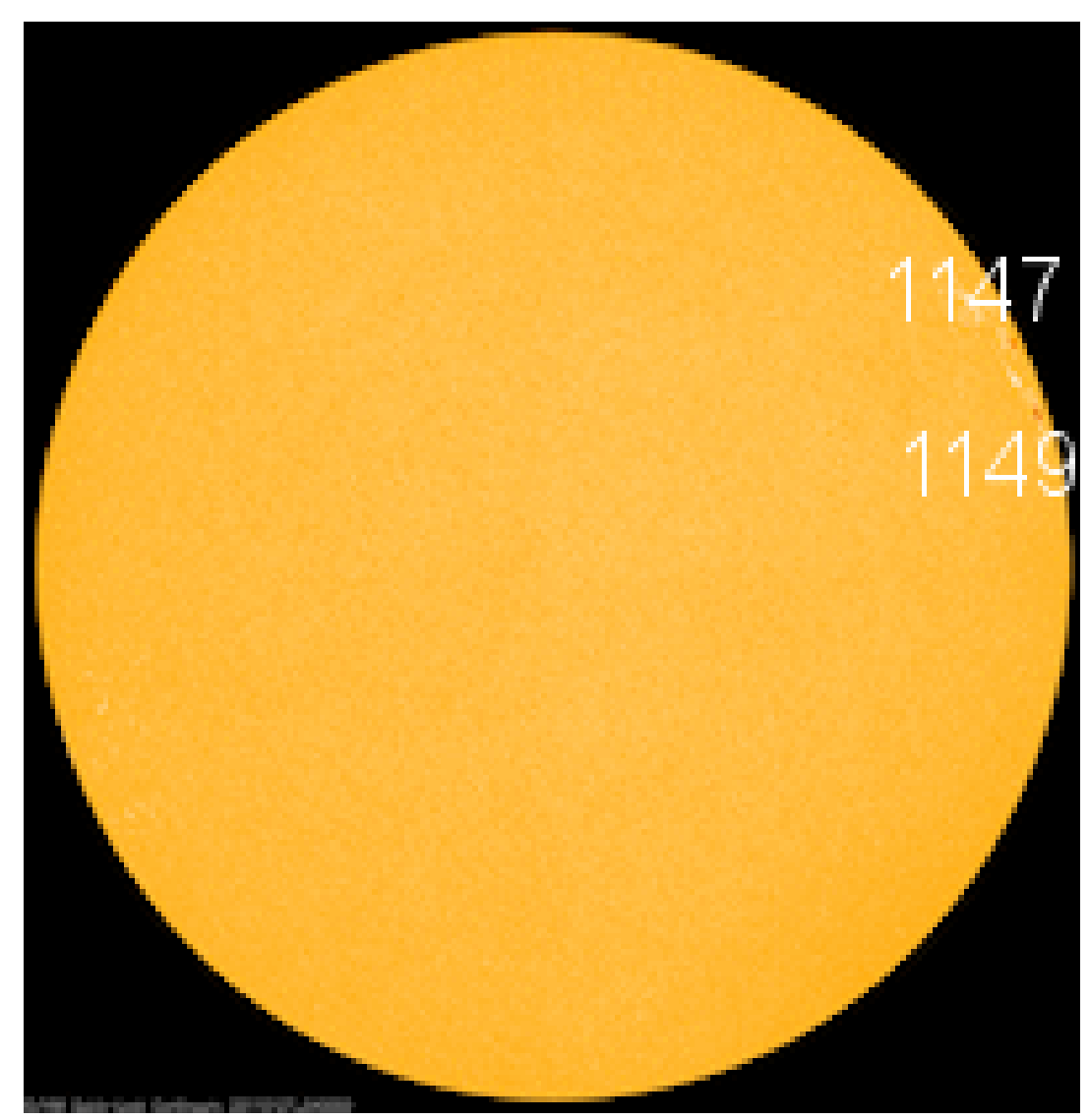

Figure 2: Departing sunspot complex 1147-1149 poses a slight threat for C-class solar flares. Credit: SDO/HMI

Based on the information provided by space weather .com, it was detected that during this time there is X-ray solar flare. It was happen due to an explosion on the sun that happen when the energy stored in twisted magnetic field which is usually occur above sunspot. X-rays and gamma rays are the sources of flare to produce a burst of radiation across the electromagnetic spectrum. Solar flare is considered as a high energetic and complicated phenomenon in which mass eruption occur. During flares, large scale of magnetic field structures can be destabilized and be repelled into the interplanetary medium; along with the large mass it contains to form so-called CMEs. Table 1 shows the condition of the Sun during $27^{\text {th }}$ January 2011.

Table 1: Space weather condition at 27th January 2011

\begin{tabular}{|l|l|}
\hline Solar Wind & X-ray Solar Flares \\
\hline speed: $\mathbf{3 1 7 . 2} \mathrm{km} / \mathrm{sec}$ & 6-hr max: C1 2003 UT Jan27 \\
density: $3.9 \mathrm{protons} / \mathrm{cm}^{3}$ & 24-hr: C1 2003 UT Jan27 \\
\hline
\end{tabular}



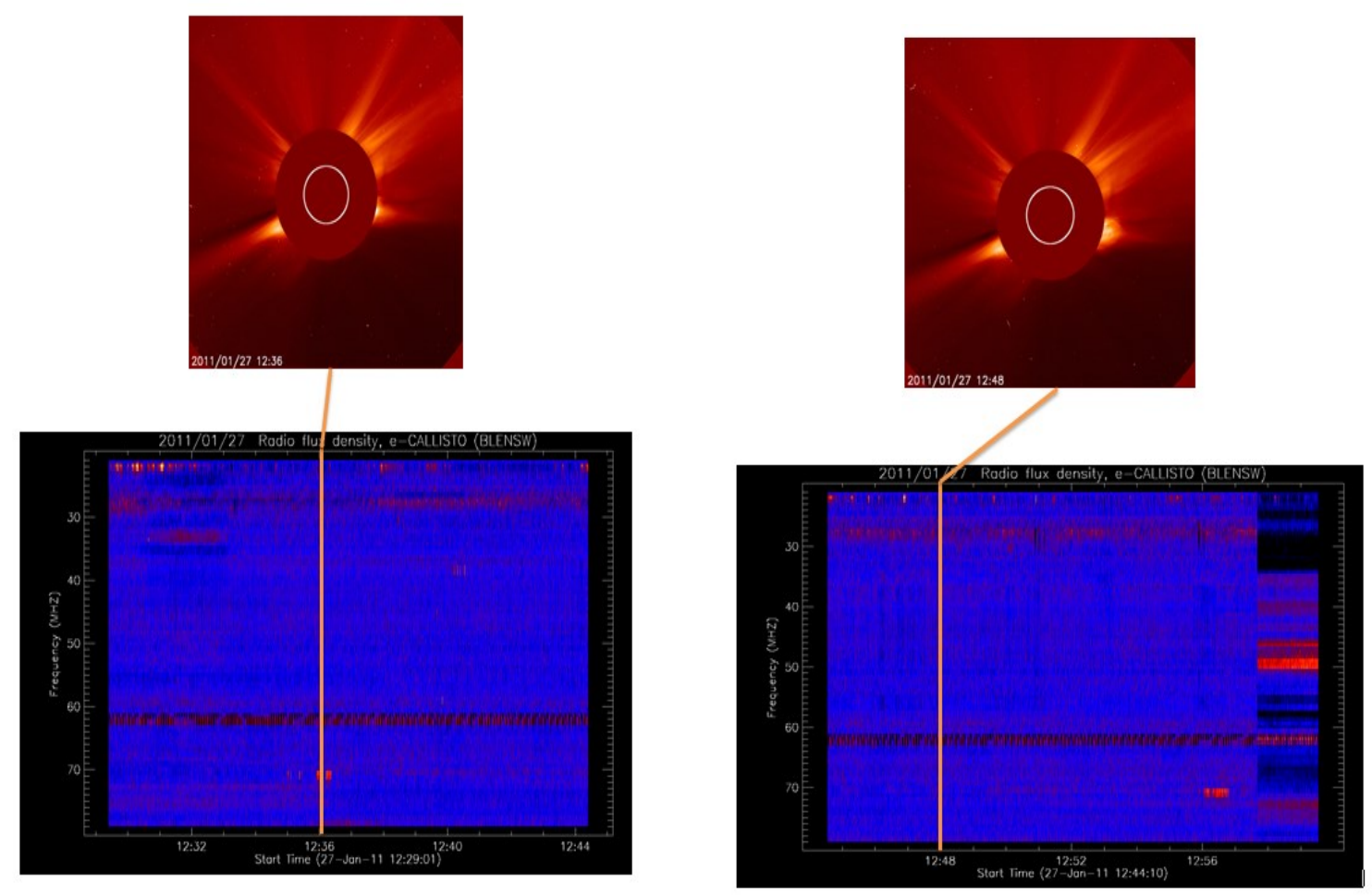

Figure 3: e-CALLISTO at 12:29 UT - 12: 45 UT and LASCO C2 images and e-Callisto at 12:44 UT - 13:00 UT and LASCO 2 images (Credited to e- Callisto and SOHO)

The observation was made till 13:00 UT. The results show that, a small loop was observed. This loop indicates that, a small CME was appearing in this time. Whereby, CMEs associated with metric type II burst and CMEs associated with IP type II burst and it is clear evidence that the energy of CME is an important factor in deciding whether it will associated with a type II burst.

\section{CONCLUDING REMARKS}

Based on the result obtained, the SRBT III is followed by SRBT II which only in short period. During the SRBT II, the solar flare was also appearing and same goes to the CMEs. During the last two decades it become clear that solar conditions greatly affect the earth and environment. Thus, it is important to observe the activities on the sun continuously to understand the unique behavior of the sun from time to time.

\section{Acknowledgement}

We are grateful to CALLISTO network; STEREO, LASCO, SDO/AIA, NOAA and SWPC make their data available online. This work was partially supported by the 600-RMI/FRGS 5/3 (135/2014), 600-RMI/RACE 16/6/2(4/2014and 600- RMI/RAGS 5/3 (121/2014) UiTM grants, Universiti Teknologi MARA and Kementerian Pendidikan Malaysia. Special thanks to the National Space Agency and the National Space Centre for giving us a site to setup this project and support this project. Solar burst monitoring is a project of cooperation between the Institute of Astronomy, ETH Zurich, and FHNW Windisch, Switzerland, MARA University of Technology and University of Malaya. The research has made use of the National Space Centre Facility and a part of an initiative of the International Space Weather Initiative (ISWI) program. 


\section{Biography}

Dr Zety Sharizat Hamidi is currently a senior lecturer and focused in Solar Astrophysics research specifically in radio astrophysics at the School of Physics and Material Sciences, Faculty of Sciences, MARA University of Technology, 40450, Shah Alam, Selangor, Malaysia. Involve a project under the International Space Weather Initiative (ISWI) since 2010.

Marhana Omar Ali is an undergraduate Physics student at the School of Physics and Material Sciences, Faculty of Sciences, MARA University of Technology, 40450, Shah Alam, Selangor, Malaysia.

Dr Nur Nafhatun Md Shariff is a senior lecturer in Academy of Contemporary Islamic Studies (ACIS), MARA University of Technology, 40450, Shah Alam, Selangor, Malaysia.Her current research is more on sustainability; environmental aspect. She is looking forward for cross-field research, i.e. solar astrophysics, light pollution measurement (mapping) and religious studies.

C.Monstein is a senior Engineer at Institute of Astronomy, Wolfgang-Pauli-Strasse 27, Building HIT, Floor J, CH-8093 Zurich, Switzerland and one of the researchers who initiated the CALLISTO system around the world.

\section{References}

[1] Z. Hamidi, Z. Abidin, Z. Ibrahim, C. Monstein, N. Shariff, M. Sabaghi, The Beginning Impulsive of Solar Burst Type IV Radio Emission Detection Associated with M Type Solar Flare, International Journal of Fundamental Physical Sciences 2 (2012).

[2] Z. Hamidi, N. Shariff, C. Monstein, Disturbances of Solar Eruption From Active Region AR1613, (2014).

[3] Z. Hamidi, N. Shariff, Detailed Investigation of a Moving Solar Burst Type IV Radio Emission in on Broadband Frequency, International Letters of Chemistry, Physics and Astronomy 7 (2014) 30-36.

[4] Z. Hamidi, Z. Abidin, Z. Ibrahim, C. Monstein, N. Shariff, Signal Detection Performed by Log Periodic Dipole Antenna (LPDA) in Solar Monitoring, International Journal of Fundamental Physical Sciences (2012).

[5] H. Aurass, Coronal Physics from Radio and Space Observations, in: I.G. Trottet (Ed.), Lecture Notes in Physics, Springer, Berlin, 1997.

[6] C.W. Young, C.L. Spencer, G.E. Moreton, J.A. and Roberts, Astrophys. J. 133 (1961).

[7] Ellis, Australian J.Phys. 22 (1969) 167.

[8] D.J. McLean, a.N.R. Labrum, Solar Radiophysics, Cambridge University Press, Cambridge, 1985.

[9] J.P. Wild, Smerd S.F., and Weiss, A.A., Solar Burst, Ann. Rev. Astron. Astrophysics 1 (1963) 291-366.

[10] Z. Hamidi, N. Shariff, The Propagation of An Impulsive Coronal Mass Ejections (CMEs) due to the High Solar Flares and Moreton Waves, (2014).

[11] Z. Hamidi, U. Ibrahim, U.F. Salwa, Z. Abidin, Z. Ibrahim, N. Shariff, Theoretical Review of Solar Radio Burst III (SRBT III) Associated With of Solar Flare Phenomena, International Journal of Fundamental Physical Sciences 3 (2013). 
[12] Z. Hamidi, N. Shariff, C. Monstein, First Light Detection of A Single Solar Radio Burst Type III Due To Solar Flare Event, (2014).

[13] G.A. Dulk, Type III solar radio bursts at long wavelengths, in: R. Stone, E. Weiler, M. Goldstein (Eds.), Geophys. Monogr., 2000.

[14] G. Swarup, P.H. Stone, A. Maxwell, ApJ 131 (1960).

[15] Z. Hamidi, N. Shariff, C. Monstein, Scenario of Solar Radio Burst Type III During Solar Eclipse on 14 th November 2012, (2014).

[16] G.B.a.L. Gelfreikh, B. I. , Soviet Astron. 23 (1979).

[17] P. Lantos, Sol. Phys. 22 (1972).

[18] A. Vourlidas, Bastian, T. S., Nitta, N., and Aschwanden, M. J., Sol. Phys. 163 (1996).

[19] E.Y. Zlotnik, Soviet Astron. 12 (1968).

[20] V.V. Zheleznyakov, Radio Emission of the Sun and Planets (1970).

[21] Z. Hamidi, N. Shariff, C. Monstein, W.W. Zulkifli, M. Ibrahim, N. Arifin, N. Amran, An Xray Observations of A Gradual Coronal Mass Ejections (CMEs) on 15th April 2012, International Letters of Chemistry, Physics and Astronomy 8 (2014) 13-19.

[22] Z. Hamidi, N. Shariff, C. Monstein, Statistical Study of Nine Months Distribution of Solar Flares, (2014).

[23] S.M. White, Solar Radio Bursts and Space Weather, Asian Journal of Physics 16 (2007) 189207.

[24] Z.S. Hamidi, N.N.M. Shariff, Chronology of Formation of Solar Radio Burst Types III and V Associated with Solar Flare Phenomenon on 19th September 2011 International Letters of Chemistry, Physics and Astronomy 5 (2014) 32-42.

[25] Z.S. Hamidi, N.N.M. Shariff, M.F. Ali, C. Monstein, W.N.A.W. Zulkifli, M.B. Ibrahim, N.S. Arifin, N.A. Amran, The Correlation between Solar Flare Phenomena in an X-ray Region and Radio Flux Measurement from January to September 2010, International Letters of Chemistry,Physics \& Astronomy 9 (2014) 84-92.

[26] Z.S. Hamidi, N.N.M. Shariff, F.N.Z. Ulum, Z.Z. Abidin, Z.A. Ibrahim, Time Series Analysis of Variance of Sunspots in January till September 2010 and Correlation with Sunspots Areas International Journal of Astronomy 5 (2012) 101-106.

[27] Z. Hamidi, Probability of Solar Flares Turn Out to Form a Coronal Mass Ejections Events Due to the Characterization of Solar Radio Burst Type II and III, International Letters of Chemistry, Physics and Astronomy 16 (2014) 85.

[28] Z. Hamidi, C. Monstein, N. Shariff, Radio Observation of Coronal Mass Ejections (CMEs) Due to Flare Related Phenomenon on 7 th March 2012, (2012).

[29] Z. Hamidi, N. Shariff, Enormous Eruption of 2.2 X-class Solar Flares on 10 th June 2014, (2014).

[30] Z. Hamidi, N. Shariff, C. Monstein, Understanding Climate Changes in Malaysia Through Space Weather Study, International Letters of Natural Sciences (2014).

[31] Z. Hamidi, N. Shariff, The Mechanism of Signal Processing of Solar Radio Burst Data in ECALLISTO Network (Malaysia), International Letters of Chemistry, Physics and Astronomy (2014).

[32] Z. HAMIDI, N. SHARIFF, Z. ABIDIN, Z. IBRAHIM, C. MONSTEIN, E-Callisto Collaboration: Some Progress Solar Burst Studies Associated with Solar Flare Research Status in Malaysia, Malaysian Journal of Science and Technology Studies 9 (2013) 15-22. 
[33] Z.S. Hamidi, N. Shariff, Z. Abidin, Z. Ibrahim, C. Monstein, Coverage of Solar Radio Spectrum in Malaysia and Spectral Overview of Radio Frequency Interference (RFI) by Using CALLISTO Spectrometer from 1MHz to $900 \mathrm{MHz}$, Middle-East Journal of Scientific Research 12 (2012) 6.

[34] Z.S.Hamidi, N.N.M.Shariff, Evaluation of Signal to Noise Ratio (SNR) of Log Periodic Dipole Antenna (LPDA) Business Engineering and Industrial Applications Colloquium 2013, IEEE, Langkawi, Malaysia, 2013, pp. 434-438.

[35] Z. Hamidi, N. Shariff, Determination of Flux Density of the Solar Radio Burst Event by Using Log Periodic Dipole Antenna (LPDA), International Letters of Chemistry, Physics and Astronomy 7 (2014) 21-29.

[36] Z. Hamidi, N. Shariff, C. Monstein, The Different Between the Temperature of the Solar Burst at the Feed Point of the Log Periodic Dipole Antenna (LPDA) and the CALLISTO Spectrometer, (2014).

[37] N. Hashim, Z. Abidin, U. Ibrahim, R. Umar, M. Hassan, Z. Rosli, Z. Hamidi, Z. Ibrahim, Radio Astronomy in Malaysia: Current Status and Outreach Activities, Astronomical Society of the Pacific Conference Series, 2011, pp. 355.

[38] Z.S. Hamidi, Z. Ibrahim, Z. Abidin, M. Maulud, N. Radzin, N. Hamzan, N. Anim, N. Shariff, Designing and Constructing Log Periodic Dipole Antenna to Monitor Solar Radio Burst: eCallisto Space Weather, International Journal of Applied Physics and Mathematics 2 (2011) 3.

[39] Z. Hamidi, N. Shariff, C. Monstein, Evaluation of Spectral Overview and Radio Frequency Interference (RFI) Sources at Four Different Sites in CALLISTO Network at the Narrow Band Solar Monitoring Region, (2014).

[40] Z.S. Hamidi, N.N.M. Shariff, C. Monstein, Z.A. Ibrahim, Space Weather: The Significance of e-CALLISTO (Malaysia) As One of Contributor of Solar Radio Burst Due To Solar Activity, International Letters of Chemistry, Physics and Astronomy 7 (2014) 37-44.

[41] Z.S. Hamidi, N.N.M. Shariff, Z.A. Ibrahim, Z.Z. Abidin, SOLAR STUDIES IN RADIO EMISSION AND OPTICAL PHOTOMETRY, University of Malaya Publisher, 2013.

[42] Z.S.Hamidi, S. Chumiran, A. Mohamad, N. Shariff, Z. Ibrahim, N. Radzin, N. Hamzan, N. Anim, A. Alias, Effective temperature of the sun based on log periodic dipole antenna performance in the range from $45 \mathrm{Mhz}$ to $870 \mathrm{Mhz}$, American Journal of Modern Physics 2 (2013) 4.

[43] Z.S.Hamidi, Z. Abidin, Z. Ibrahim, N. Shariff, C. Monstein, Modification and Performance of Log Periodic Dipole Antenna, International Journal of Engineering Research and Development 3 (2012) 36-39.

[44] Z.S.Hamidi, Z. Abidin, Z. Ibrahim, C. Monstein, N. Shariff, Signal Detection Performed by Log Periodic Dipole Antenna (LPDA) in Solar Monitoring, International Journal of Fundamental Physical Sciences 2 (2012) 32-34.

[45] Z.S.Hamidi, N.M.Anim, N. N.S.Hakimi, N.Hamzan, A.Mokhtar, N.Syukri, S.Rohizat, I.Sukma, Z.A. Ibrahim, Z.Z.Abidin, N.N.M.Shariff, C.Monstein, Application of Log Periodic Dipole Antenna (LPDA) in Monitoring Solar Burst at Low Region Frequencies Region International Journal of Fundamental Physical Sciences 2 (2012) 4.

[46] Z.S.Hamidi, N.N.M. Shariff, Determination of Isotropic Source Spectral Power of the Log Periodic Dipole Antenna (LPDA), International Journal of Science and Mathematics 2 (2014) 3. 
[47] Z.S.Hamidi, Z. Abidin, Z. Ibrahim, N. Shariff, Indication of radio frequency interference (RFI) sources for solar burst monitoring in Malaysia, ICPAP 2011, AIP Publisher, Indonesia, 2012, pp. 6.

[48] Z.S. Hamidi, N.N.M. Shariff, C. Monstein, High Time Resolution Observation of Solar Radio of A Group Type III And U Burst Associated of Solar Flares Event, The International Journal of Engineering 1 (2012) 3.

[49] Z. Hamidi, N. Shariff, C. Monstein, W.W. Zulkifli, M. Ibrahim, N. Arifin, N. Amran, Observation of the Radio Frequency Interference (RFI) at the National Space Centre, Malaysia, International Letters of Natural Sciences (2014).

[50] Z.S. Hamidi, Z.Z. Abidin, Z.A. Ibrahim, N.N.M. Shariff, U.F.S.U. Ibrahim, R. Umar, Preliminary analysis of investigation Radio Frequency Interference (RFI) profile analysis at Universiti Teknologi MARA, IEEE, 2011, pp. 311-313.

[51] R. Umar, Z. Abidin, Z. Ibrahim, N. Gasiprong, K. Asanok, S. Nammahachak, S. Aukkaravittayapun, P. Somboopon, A. Prasit, N. Prasert, The Study of Radio Frequency Interference (RFI) in Altitude Effect on Radio Astronomy In Malaysia And Thailand, Middle East Journal of Scientific Research 14 (2013).

[52] Z.S.Hamidi, N.N.M.Shariff, R.Umar, Influence Factors of Radio Frequency Interference (RFI) for Solar Radio Astronomy Purpose at National Space Centre, Malaysia Thailand Journal of Physics 3 (2012) 6.

[53] R. Umar, Z.Z. Abidin, Z.A. Ibrahim, M.S.R. Hassan, Z. Rosli, Z.S. Hamidi, Population density effect on radio frequencies interference (RFI) in radio astronomy, AIP Conference Proceedings 1454 (2012) 39.

[54] N. Anim, Z. Hamidi, Z. Abidin, C. Monstein, N. Rohizat, Radio frequency interference affecting type III solar burst observations, 2012 NATIONAL PHYSICS CONFERENCE:(PERFIK 2012), American Institute of Physics, 2013, pp. 82-86.

[55] Z. Hamidi, N. Shariff, C. Monstein, Comparison of the Radio Frequency Interference (RFI) in the Region of Solar Burst Type III Data At Selected CALLISTO Network, International Letters of Chemistry, Physics and Astronomy 10 (2014) 38-45. 